\title{
ON A $q$-ANALOGUE OF THE MULTIPLE GAMMA FUNCTIONS
}

\author{
MICHITOMO NISHIZAWA
}

\begin{abstract}
A $q$-analogue of the multiple gamma functions is introduced, and is shown to satisfy the generalized Bohr-Morellup theorem. Furthermore we give some expressions of these function.
\end{abstract}

1995 Mathematical Subject Classification. 33D05, 81R50 


\section{INTRODUCTION}

In 1904, E.W.Barnes defined the multiple gamma functions relevant to his zeta functions [2], [3], [4], [5]. His works were influenced by the study of the integral functions which are motivated by number theory. M.F.Vigneŕas investigated the multiple gamma functions on her paper 19. She showed these functions to satisfy the "generalized Bohr-Morellup theorem". These functions have been studied by many authors relevant to the determinant of Laplacian on Riemann surfaces and the Selberg zeta function in number theory and physics (For example [18], [20]). On the other hand, the $q$-gamma function was defined by F.H.Jackson [8], 9]. R.Askey showed these function satisfies a $q$ analogue of the Bohr-Morellup theorem [1]. Recently P.G.O.Freund and A.V.Zabrodin constructed a hierarchy of S-matrices of integrable models by using the multiple gamma function and its $q$-analogue [7]. But their $q$-analogue remains some ambiguities because there is not the definition of the normalization factor of these functions in their paper. Motivated by Vigneŕas' and Askey's works, we construct a $q$-analogue of the multiple gamma functions exactly, which is a generalization of the $q$-gamma function and satisfies a $q$-analogue of the generalized Bohr-Morellup theorem. We can see these functions have some expressions like the gamma function. Furthermore, they are related to a $q$-multiple zeta functions like the case of Barnes' multiple gamma function and his multiple zeta functions [16], [17].

The author expresses his deep gratitude Professor Kimio Ueno for helpful discussions and constant encouragement.

\section{A SURVEy OF THE MULTiPle GAMma FUNCTiONS}

In this section we give a brief survey of the basic facts concerning the gamma function and its generalization.

The gamma function are characterized Bohr-Morellup theorem.

Theorem 2.1 (Bohr-Mollerup). The gamma function satisfies

1. $\Gamma(z+1)=z \Gamma(z)$,

2. $\Gamma(1)=1$,

3. $\frac{d^{2}}{d z^{2}} \log \Gamma(z+1) \geq 0$ for $z \geq 0$,

and the function satisfying (1),(2),(3) is determined uniquely.

We use expression of this function as follows.

$$
\Gamma(z+1)=\lim _{N \rightarrow \infty} \frac{N !}{(z+1)(z+2) \cdots(z+N)}(N+1)^{z} .
$$




$$
\begin{gathered}
\Gamma(z+1)=\prod_{n=1}^{\infty}\left\{\left(1+\frac{1}{n}\right)^{z}\left(1+\frac{z}{n}\right)^{-1}\right\} . \\
\Gamma(z+1)=e^{-\gamma x} \prod_{n=1}^{\infty}\left\{\left(1+\frac{z}{n}\right)^{-1} e^{\frac{z}{n}}\right\},
\end{gathered}
$$

where $\gamma$ is Euler constant.

As generalization of the gamma function, M.F.Vignéras constructed the multiple gamma functions by using a generalization of the BohrMorellup theorem [19]. First, we remark a theorem due to Dufresnoy and Pisot [6].

Theorem 2.2 (Dufresnoy and Pisot). Let $g(z)$ be a $k$ times differentiable function and $g^{(k)}(z) \rightarrow 0$ as $z \rightarrow \infty$, then the function $f(z)$ which satisfies

$$
f(z+1)-f(z)=g(z)
$$

exists. It is unique if $f(0)$ is given. Furthermore, $f(z)$ is $k$ times differentiable and $f^{(k)}(z)$ is increasing for $x \geq 0$

By this theorem, if we put

$$
f_{0}(z)=\log (z+1)
$$

then we can determine $f_{r}(z)$ to satisfy

$$
f_{r}(z)-f_{r}(z-1)=f_{r-1}(z), \quad f_{r}(0)=0,
$$

because $f_{r}(z)$ is $(r+1)$-times differentiable and $f_{r}^{(r+1)}(z) \rightarrow 0$ as $z \rightarrow$ $\infty$. Hence we can define

$$
G_{r}(z+1)=\exp f_{r}(z)
$$

then the next theorem follows (cf.[19]).

Theorem 2.3. The functions $G_{r}(z)$ satisfies

(1) $G_{r}(z+1)=G_{r-1}(z) G_{r}(z)$

(2) $G_{r}(1)=1$,

(3) $\frac{d^{r+1}}{d z^{r+1}} \log G_{r}(z+1) \geq 0 \quad$ for $\quad z \geq 0$,

(4) $G_{0}(z)=z$.

And meromorphic function satisfies above properties is determined uniquely.

These functions are called "the multiple gamma functions". For example, $G_{1}(z)$ is $\Gamma(z)$. 


\section{The $q$-Gamma FUnCtion}

In this section we review the $q$-analogue of the gamma function (The $q$-gamma function), which is known as

$$
\Gamma(z+1 ; q)=(1-q)^{-z} \prod_{n=1}^{\infty}\left(\frac{1-q^{z+n}}{1-q^{n}}\right)^{-1} .
$$

R.Askey showed this function satisfies a $q$-analogue of the BohrMorellup theorem.

Theorem 3.1 (Askey). For $0<q<1$, the function $\Gamma(z ; q)$ satisfies

1. $\Gamma(z+1 ; q)=[z] \Gamma(z ; q)$,

2. $\Gamma(1 ; q)=1$,

3. $\frac{d^{2}}{d z^{2}} \log \Gamma(z+1 ; q) \geq 0$ for $z \geq 0$.

The uniqueness of this function follows from this theorem and theorem 2.2. This theorem suggests that Vigneras method can be applied to a $q$-analogue of the multiple gamma functions.

The $q$-gamma function has the expressions which correspond to (2.1), (2.2). It is easy to show

$$
\Gamma(z+1 ; q)=\lim _{N \rightarrow \infty} \frac{[1][2] \cdots[N]}{[z+1][z+2] \cdots[z+N]}[N+1]^{z}
$$

and

$$
\Gamma(z+1 ; q)=\prod_{n=1}^{\infty}\left\{\left(\frac{[n+1]}{[n]}\right)^{z}\left(\frac{[z+n]}{[n]}\right)^{-1}\right\},
$$

where we use a notation

$$
[z]=\frac{1-q^{z}}{1-q} .
$$

4. A q-analogue of the multiple gamma FunCtions

In this section, we define a $q$-analogue of the multiple gamma functions which satisfy a $q$-analogue of the generalized Bohr-Morellup theorem, and we derive the expressions corresponding to (2.1), (2.2). We assume $0<q<1$.

Definition 4.1. Let $z$ be in the right half plane $\{s \in \mathbf{C} \mid \Re \mathbf{s}>\mathbf{0}\}$ and $r \in \mathbf{Z}_{\geq 0}$, we define

$$
\begin{gathered}
G_{0}(z+1 ; q):=[z+1], \\
G_{r}(z+1 ; q):=(1-q)^{-\left(\begin{array}{c}
z \\
r
\end{array}\right)} \prod_{n=1}^{\infty}\left\{\left(\frac{1-q^{z+n}}{1-q^{n}}\right)^{(-1)^{r}\left(\begin{array}{c}
n+r-2 \\
r-1
\end{array}\right)}\left(1-q^{n}\right)^{g_{r}(z, n)}\right\}, \quad \text { for } \quad r \geq 1
\end{gathered}
$$


where

$g_{1}(z, n):=0, \quad g_{r}(z, n):=\sum_{m=1}^{r-1}(-1)^{m-1}\left(\begin{array}{c}z \\ r-m\end{array}\right)\left(\begin{array}{c}n+m-2 \\ m-1\end{array}\right) \quad$ for $\quad r \geq 2$

For example, $G_{1}(z ; q)$ is $\Gamma(z ; q)$. The infinite products of these functions are absolutely convergent, First, we prove a $q$-analogue of Theorem 2.3

Theorem 4.2. If $\Re z>0$, then $G_{r}(z: q)$ satisfy

1. $G_{r}(z+1 ; q)=G_{r-1}(z ; q) G_{r}(z ; q)$,

2. $G_{r}(1 ; q)=1$,

3. $\frac{d^{r+1}}{d z^{r+1}} \log G_{r+1}(z+1 ; q) \geq 0$ for $z \geq 0$,

4. $G_{0}(z ; q)=[z]$.

The functions satisfying such properties are determined uniquely.

Proof. First we remark next formulas.

Lemma 4.3. $\quad$ 1. $g_{r}(0, n)=0$.

2. $g_{r}(z, n)-g_{r}(z-1 . n)=g_{r-1}(z-1, n)-(-1)^{r-1}\left(\begin{array}{c}n+r-3 \\ r-2\end{array}\right)$.

These formula can be proved by direct calculation. Next we prove the claim of theorem. When $r=1$, the claims are Theorem 3.1. So it is sufficient to show the case $r \geq 2$. (2),(4) can be proved easily. So we prove (1) and (3).

(1)We can see

$$
\begin{aligned}
& G_{r}(z+1 ; q) \\
&=(1-q)^{-\left(\begin{array}{c}
z-1 \\
r
\end{array}\right)-\left(\begin{array}{c}
z-1 \\
r-1
\end{array}\right)} \prod_{n=1}^{\infty}\left\{\left(1-q^{z+n-1}\right)^{(-1)^{r}\left(\begin{array}{c}
n+r-2 \\
r-1
\end{array}\right)-(-1)^{r}\left(\begin{array}{c}
n+r-3 \\
r-1
\end{array}\right)}\right. \\
& \quad \times\left(1-q^{n}\right)^{\left.-(-1)^{r}\left(\begin{array}{c}
n+r-2 \\
r-1
\end{array}\right)+g_{r}(z-1, n)+g_{r-1}(z-1, n)-(-1)^{r-1}\left(\begin{array}{c}
n+r-3 \\
r-2
\end{array}\right)\right\}} \\
&=(1-q)^{-\left(\begin{array}{c}
z-1 \\
r-1
\end{array}\right)} \prod_{n=1}^{\infty}\left\{\left(\frac{1-q^{z+n-1}}{1-q^{n}}\right)^{(-1)^{r-1}\left(\begin{array}{c}
n+r-3 \\
r-2
\end{array}\right)}\left(1-q^{n}\right)^{g_{r-1}(z-1, n)}\right\} \\
& \quad \times(1-q)^{-\left(\begin{array}{c}
z-1 \\
r
\end{array}\right)} \prod_{n=1}^{\infty}\left\{\left(\frac{1-q^{z+n-1}}{1-q^{n}}\right)^{(-1)^{r}\left(\begin{array}{c}
n+r-2 \\
r-1
\end{array}\right)}\left(1-q^{n}\right)^{g_{r}(z-1, n)}\right\} \\
&=G_{r-1}(z ; q) G_{r}(z ; q) .
\end{aligned}
$$

(3) We can see

$$
\frac{d^{r+1}}{d z^{r+1}} \log G_{r}(z+1 ; q)
$$




$$
\begin{aligned}
& =(-1)^{r+1} \frac{d^{r+1}}{d z^{r+1}}\left\{\sum_{n=1}^{\infty} \sum_{k=1}^{\infty}\left(\begin{array}{c}
n+r-2 \\
r-1
\end{array}\right) \frac{q^{(z+n) k}}{k}\right\} \\
& =(-\log q)^{r+1} \sum_{n=1}^{\infty} \sum_{k=1}^{\infty}\left(\begin{array}{c}
n+r-2 \\
r-1
\end{array}\right) k^{r} q^{(z+n) k} \\
& \geq 0,
\end{aligned}
$$

because $\log q<0$.

Finally, we prove the uniqueness of these functions. Because of the formula in the proof (3),

$$
\frac{d^{r+1}}{d z^{r+1}} \log G_{r}(z+1 ; q) \rightarrow 0
$$

as $z \rightarrow \infty$. Hence the uniqueness follows from Theorem 2.2.

By Theorem $4.2(1), G_{r}(z ; q)$ can be defined when $z \neq l+m \log q / 2 \pi$ $(l$; negative integer, $m \in \mathbf{Z})$. Next we consider expression like the formulas (2.1), (2.2).

Proposition 4.4. If $z z>0$, then

$$
\begin{aligned}
& G_{r}(z+1 ; q)=\lim _{N \rightarrow \infty}\left\{\frac{G_{r-1}(1 ; q) \cdots G_{r-1}(N ; q)}{G_{r-1}(z+1 ; q) \cdots G_{r-1}(z+N ; q)} \prod_{m=1}^{r} G_{r-m}(N+1 ; q)^{\left(\begin{array}{c}
z \\
m
\end{array}\right)}\right\} \\
& G_{r}(z+1 ; q)=\prod_{n=1}^{\infty}\left\{\frac{G_{r-1}(n ; q)}{G_{r-1}(z+n ; q)} \prod_{m=1}^{r}\left(\frac{G_{r-m}(n+1 ; q)}{G_{r-m}(n ; q)}\right)^{\left(\begin{array}{c}
z \\
m
\end{array}\right)}\right\} .
\end{aligned}
$$

Proof. (1) First we prove next formulas

Lemma 4.5. (1) For $r \geq 1$

$$
G_{r}(N+1 ; q)=(1-q)^{-\left(\begin{array}{c}
N \\
r
\end{array}\right)} \prod_{n=1}^{N}\left(1-q^{n}\right)^{\left(\begin{array}{c}
N-n \\
r-1
\end{array}\right) .}
$$

(2) For $r \geq 2, N \geq 1$

$$
\sum_{m=1}^{r-1}\left(\begin{array}{c}
z \\
m
\end{array}\right)\left(\begin{array}{c}
N \\
r-m
\end{array}\right)=\sum_{n=1}^{N}\left\{\left(\begin{array}{c}
z+n-1 \\
r-1
\end{array}\right)-\left(\begin{array}{c}
n-1 \\
r-1
\end{array}\right)\right\} .
$$

(3) For $N \geq 1, n \geq 1, r \geq 2$,

$$
\sum_{m=1}^{r+1}\left(\begin{array}{c}
N-n \\
r-m
\end{array}\right)\left(\begin{array}{c}
z \\
m
\end{array}\right)-\sum_{k=1}^{N}\left\{g_{r}(z+k-1, n)-g_{r}(k-1, n)\right\}=g_{r+1}(z, n) .
$$


ON A $q$-ANALOGUE OF THE MULTIPLE GAMMA FUNCTIONS $\quad 7$

They are shown by induction. We prove (1) of Proposition 4.4. By Lemma 4.5 (1),

$$
\begin{aligned}
& \lim _{N \rightarrow \infty}\left\{\frac{G_{r-1}(1 ; q) \cdots G_{r-1}(N ; q)}{G_{r-1}(z+1 ; q) \cdots G_{r-1}(z+N ; q)} \prod_{m=1}^{r} G_{r-m}(N+1 ; q)^{\left(\begin{array}{c}
z \\
m
\end{array}\right)}\right\} \\
& =\lim _{N \rightarrow \infty}\left\{(1-q)^{\sum_{k=1}^{N}\left\{\left(\begin{array}{c}
z+k-1 \\
r-1
\end{array}\right)-\left(\begin{array}{c}
k-1 \\
r-1
\end{array}\right)\right\}-\sum_{m=1}^{r-1}\left(\begin{array}{c}
N \\
r-m
\end{array}\right)\left(\begin{array}{c}
z \\
m
\end{array}\right)-\left(\begin{array}{c}
z \\
r
\end{array}\right)} \times \prod_{k=1}^{N} \prod_{n=1}^{\infty}\left(\frac{1-q^{z+n+k-1}}{1-q^{n+k-1}}\right)^{(-1)^{r}\left(\begin{array}{c}
n+r-3 \\
r-2
\end{array}\right)}\right. \\
& \quad \times \prod_{n=1}^{N}\left(1-q^{n}\right)^{\sum_{m=1}^{r}\left(\begin{array}{c}
N-n \\
r-m
\end{array}\right)\left(\begin{array}{c}
z \\
m
\end{array}\right)-\sum_{k=1}^{N}\left\{g_{r-1}(z+k-1, n)-g_{r-1}(k-1, n)\right\}} \\
& \left.\quad \times \prod_{n=N+1}^{\infty}\left(1-q^{n}\right)^{-\sum_{k=1}^{N}\left\{g_{r-1}(z+k-1, n)-g_{r-1}(k-1, n)\right\}}\right\} .
\end{aligned}
$$

By Lemma 4.5 (2), (3) and

$$
\prod_{k=0}^{\infty} \prod_{n=1}^{\infty}\left(\frac{1-q^{z+n+k-1}}{1-q^{n+k-1}}\right)^{(-1)^{r}\left(\begin{array}{c}
k+r-3 \\
r-2
\end{array}\right)}=\prod_{n=1}^{\infty}\left(\frac{1-q^{z+n}}{1-q^{n}}\right)^{(-1)^{r}\left(\begin{array}{c}
n+r-2 \\
r-1
\end{array}\right)},
$$

we obtain

$$
\begin{aligned}
& \lim _{N \rightarrow \infty}\left\{\frac{G_{r-1}(1 ; q) \cdots G_{r-1}(N ; q)}{G_{r-1}(z+1 ; q) \cdots G_{r-1}(z+N ; q)} \prod_{m=1}^{r} G_{r-1}(N+1 ; q)^{\left(\begin{array}{c}
z \\
m
\end{array}\right)}\right\} \\
& =\left\{(1-q)^{-\left(\begin{array}{c}
z \\
r
\end{array}\right)} \prod_{n=1}^{\infty}\left\{\left(\frac{1-q^{z+n}}{1-q^{n}}\right)^{(-1)^{r}\left(\begin{array}{c}
n+r-2 \\
r-1
\end{array}\right)}\left(1-q^{n}\right)^{g_{r}(z, n)}\right\}\right\} \\
& \times \lim _{N \rightarrow \infty}\left\{\prod_{n=N+1}^{\infty}\left(1-q^{n}\right)^{-\sum_{m=1}^{r}\left(\begin{array}{c}
N-n \\
r-m
\end{array}\right)\left(\begin{array}{c}
z \\
m
\end{array}\right)}\right\} .
\end{aligned}
$$

So, it is sufficient to show

$$
\prod_{n=N+1}^{\infty}\left(1-q^{n}\right)^{\left(\begin{array}{c}
N-n \\
r-m
\end{array}\right)\left(\begin{array}{c}
z \\
m
\end{array}\right)} \rightarrow 1 \quad \text { as } \quad N \rightarrow \infty .
$$

We can see

$$
\begin{aligned}
& \left|\log \left\{\prod_{n=N+1}^{\infty}\left(1-q^{n}\right)\left(\begin{array}{c}
N-n \\
r-m
\end{array}\right)\left(\begin{array}{c}
z \\
m
\end{array}\right)\right\}\right| \leq\left|\left(\begin{array}{c}
z \\
m
\end{array}\right)\right| \sum_{n=N+1}^{\infty}\left|\left(\begin{array}{c}
N-n \\
r-m
\end{array}\right)\right|\left|\log \left(1-q^{n}\right)\right| \\
& \leq \frac{\left|\left(\begin{array}{c}
z \\
m
\end{array}\right)\right|}{(r-m) !(1-q)} \sum_{n=N+1}^{\infty}(n-N)(n-N+1) \cdots(n-N+r-m-1) q^{n} \\
& =q^{N}\left\{\frac{\left|\left(\begin{array}{c}
z \\
m
\end{array}\right)\right|}{(r-m) !(1-q)} \sum_{n=1}^{\infty}(n+r-m-1)(n+r-m-2) \cdots(n+1) \cdot n q^{n}\right\},
\end{aligned}
$$


where we take the principal value of logarithms. This tend to 0 as $N \rightarrow \infty$ on any region, since

$$
\frac{1}{(r-m) !(1-q)} \sum_{n=1}^{\infty}(n+r-m-1)(n+r-m-2) \cdots(n+1) \cdot n \cdot q^{n}
$$

takes finite value. Thus,

$$
\left|\log \left\{\prod_{n=N+1}^{\infty}\left(1-q^{n}\right)^{\left(\begin{array}{c}
N-n \\
r-m
\end{array}\right)\left(\begin{array}{c}
z \\
m
\end{array}\right)}\right\}\right| \rightarrow 0 \quad \text { as } \quad N \rightarrow \infty .
$$

Hence the claim follows.

(2) By using (1), we can prove the claim easily.

\section{REFERENCES}

[1] R.Askey, The q-Gamma and q-Beta functions Appl. Anal 8 (1978), p. 125-141.

[2] E.W.Barnes, The theory of G-function, Quat. J. Math 31 (1899), p. 264-314.

[3] _ Genesis of the double gamma function Proc. London. Math. Soc 31 (1900), p. 358-381.

[4] 196 (1900), p. 265-388.

[5] - On the theory of the multiple gamma functions, Trans. Cambridge. Phil. Soc. 19 (1904), p. 374-425.

[6] J.Dufresnoy et C.Pisot, Sur la relation fanctionalle $f(x+1)-f(x)=\phi(x)$, Bull. Soc. Math. Belgique. 15 (1963), p. 259-270.

[7] P.G.O.Freund and A.V.Zabrodin, Hierarchic array of Integrable models, Chicago preprint EFI 92-29

[8] F.H.Jackson, A generalization of the functions $\Gamma(n)$ and $x^{n}$, Proc. Roy. Soc. London. 74 (1904), p. 64-72

[9] — The basic gamma function and the elliptic functions, Proc. Roy. Soc. London. A 76 (1905), p. 127-144

[10] T.Koonwinder, Jacobi function as limit cases of q-ultraspherical polynomial, J. Math. Anal. and Appl 148 (1990), p. 44-54

[11] N.Kurokawa, Multiple sine functions and Selberg zeta functions, Proc. Japan. Acad. 67 A (1991), p 61-64

[12] _ Multiple zeta functions; an example Adv. Studies. Pure. Math. 21 (1992), p 219-226

[13] _ Gamma factors and Plancherel measures Proc. Japan. Acad. 68 A (1992), p 256-260

[14] (1992), p. $1-10$

[15] E.C.Tichmarsch, The Theory of Functions, Second edition, Oxford Univ. Press

[16] K.Ueno, private communications and unpublished notes.

[17] K.Ueno, M.Nishizawa. Quantum groups and zeta-functions Proc.of the Karpacz Winter School 1994, hep-th/9408143

[18] I.Vardi, Determinants of Laplacians and multiple gamma functions, SIAM. J. Math. Anal 19 (1988), p. 493-507. 
[19] M.F.Vignéras, L'équation fonctionalie de la fonction zeta de Selberg de groupe modulaire PSL(2,Z), Asterisque. 61 (1979), p. 235-249.

[20] A.Voros, Spectral functions, Special functions and the Selberg zeta functions, Comm. Math. Phys. 110 (1987), p. 431-465

[21] E.T.Whittacker and G.N.Watson, A Course of Modern Analysis, Fourth edition, Cambrige Univ. Press

Department of Mathematics, School of Science and Engineering, WASEDA UNIVERsity, 3-4-1 OHKubo,Shinjuku,TOKYo,JAPAN

E-mail address: 64m503@cfi.waseda.ac.jp 